\title{
Importancia de la ética y auditoría al interior de las organizaciones
}

\section{Importance of Ethics and Internal Audit of Organizations}

Yéssica María Velásquez-Herrera; Ingrid Yulmara Rojas-Arango²;

Jhon Jairo Camargo-Flechas³; Germán Fuentes-Rodríguez ${ }^{4}$

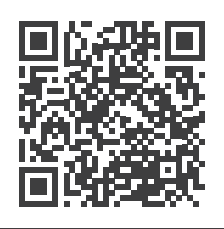

Palabras clave:

auditoría, auditor, independencia del auditor, auditoría externa, auditoría interna, auditoría pública, contabilidad, datos contables, datos de auditoría, gestión financiera, contabilidad gerencial.

Artículo de reflexión:

Fecha de recepción: 20/11/2019

Fecha de aceptación: 29/05/2020

Esta publicación se encuentra bajo licencia:

Creative Commons ReconocimientoNoComercialSinObraDerivada 4.0 Internacional

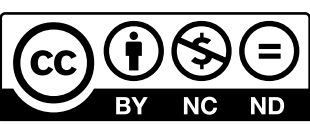

\section{Resumen}

Las Normas Internacionales de Auditoría Financiera son normas de calidad para realizar una auditoría financiera, implementadas por un auditor o revisor fiscal. La presente reseña tiene como objetivo identificar la importancia del papel del auditor con respecto a la información financiera y no financiera, mediante una investigación documental. Se identifica que una de sus principales aplicaciones es la auditoría financiera y una de las más importantes para las empresas, porque refleja la situación actual en términos cuantitativos y busca determinar si hay diferencias con los documentos auditados y no auditados. También se busca determinar qué tipo de información es financiera y cuál es la información no financiera que tiene relevancia en nuestra auditoría. Por tal motivo, el auditor debe ser demasiado cuidadoso, porque podrá encontrar congruencias con respecto a la información financiera ya auditada o a los estados financieros, y aunque no debe dar un dictamen de ello, sí es importante tenerla en cuenta para su informe final.

1 Administradora financiera (e), Corporación Universitaria Minuto de Dios, Colombia.yvelazquezh@uniminuto.edu.co, ORCID: https://orcid.org/00000001-9662-9679.

2 Administradora financiera, Corporación Universitaria Minuto de Dios, Colombia.irojasarang@uniminuto.edu.co, ORCID: https://orcid.org/00000003-2754-1280

3 Economista, magíster en Paz, Desarrollo y Ciudadanía, Corporación Universitaria Minuto de Dios, Colombia.jcamargofle@uniminuto.edu.co, ORCID: https://orcid.org/0000-0003-1832-118X

4 Administrador de empresas, magíster en Administración, Corporación Universitaria Minuto de Dios, Colombia.gfuentesrod@uniminuto.edu.co, ORCID: https://orcid.org/0000-0002-3093-1135 


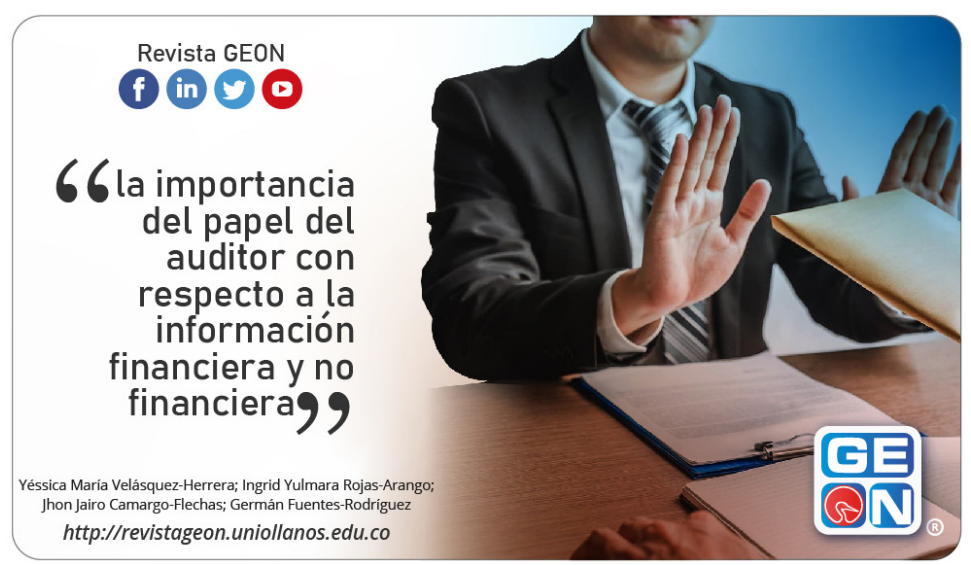

Códigos JEL: M42, M48

\section{Abstract}

The International Financial Auditing Standards are quality standards for conducting a financial audit, implemented by an auditor or statutory auditor. The purpose of this review is to identify the importance of the auditor's role regarding financial and non-financial information, through a documentary investigation. It is identified that one of its main applications is the financial audit and the most important for companies, because it reflects the current situation in quantitative terms, and seeks to determine if there are differences with the audited and unaudited documents. It also seeks to determine what type of information is financial and what is the non-financial information that is relevant in our audit, for this reason the auditor must be too careful, because he will be able to find congruence, regarding the already audited financial information or the statements financial, and although you should not give an opinion on it, it is important to take it into account for your final report.

Velasquez Herrera, Y. M., Rojas Arango, I. Y. ., Camargo Flechas, J. J., \& Fuentes Rodríguez, G. (2020). Importancia de la Etica y Auditoria al Interior de las Organizaciones. Revista GEON (Gestión, Organizaciones Y Negocios), 7(2), 1-10. https://doi. org/10.22579/23463910.198

Keywords: Audit, Auditor, Auditor independence, External audit, Internal audit, Public audit, Accounting, Accounting data, Audit data, Financial management, Managerial accounting. 


\section{Introducción}

Con la entrada en vigencia del decreto 302 de 2015, se precisa el cumplimiento de las normas internacionales de auditoría (NIA)y su implementación en Colombia a partir de 2016. Las NIA le permiten al contador público que preste sus servicios como auditor o revisor fiscal y la implementación de herramientas estandarizadas que le faciliten la revisión y aplicación en su trabajo profesional. Además, para los propietarios y accionistas de las empresas, estas normas son de gran relevancia, debido a que en el mundo financiero de hoy la reglamentación evoluciona y cambia rápidamente; es por esto por lo que las empresas deben estar dispuestas a adaptarse a estos cambios.

Se hace cada día más complejo entender por qué al interior de las organizaciones se presentan casos de fraude financiero de gran magnitud a pesar de que se ejercen controles internos y externos que fiscalizan la legalidad, constitución y situación financiera de las empresas. Es cierto que el desconocimiento de la norma no exime su responsabilidad, puesto que las actualizaciones en normas internacionales de auditoría NIA son publicadas y dadas a conocer con el fin de ser globalizadas para contrarrestar y mitigar el deterioro operativo y financiero generado cuando se omite información a nivel interno del ente económico y el impacto en el entorno socioeconómico y el mercado en general, lo que llega a afectar incluso la inversión extranjera.
Durante los últimos diez años, en Colombia se han dado a conocer escándalos de empresas reconocidas como Interbolsa, Odebrecht, Banco Agrario de Colombia, entre otros; por lo tanto, se cuestiona la eficiencia y la eficacia en los procesos y el papel tanto del contador público y/o administradores financieros como de las personas responsables de recopilar, organizar, tabular, e interpretar la información que se emite a través de su dictamen.

No cabe duda de que los desfalcos y los casos de corrupción mencionados, entre otros, han representado descalabros para la economía en general. Por esta razón, es de suma importancia que el futuro auditor, llámese contador público o revisor fiscal, actúe con imparcialidad teniendo en cuenta que con su firma da fe pública de la veracidad de la información.

\section{Contexto teórico}

Las NIA fueron creadas por la Federación Internacional de Contadores (IFAC) con el firme objetivo de evaluar la información presentada por las empresas de manera interna, dirigida a los directivos, y de manera externa, dirigida al público en general y a la compañía, con el fin de evidenciar los procesos y procedimientos que se están ejecutando, sin importar el lugar donde se encuentre la sede o sucursal de la empresa. No posee complicaciones en su información auditada, porque son normas internacionales; eso quiere decir que se aplican para todo el contexto global. Sin importar la actividad económica, tipo y clase, se 
debe realizar el mismo procedimiento en los distintos tipos de auditoría.

Según Nuño (2017), la auditoría financiera "[...] También conocida como auditoría contable, se trata de un método por el que se examina y analiza la información que una empresa tiene reflejada en los estados de sus cuentas". En otras palabras, permite vigilar los ingresos y egresos de la compañía y mitigar así las desviaciones de dinero, que, de llegar a encontrarse, podrían llevar a la quiebra a la compañía o a que tenga falta de solvencia y liquidez. Con base en lo anterior, es el departamento que requiere de más cuidado y se recomienda que el auditor sea muy sigiloso y minucioso con las auditorías.

El auditor debe encargarse de que la empresa le proporcione toda la información suficiente para poder iniciar su plan de trabajo con el objetivo de realizar sugerencias y cambios en el informe final de la auditoría. De acuerdo con Roberto (2019), "[...] Lo que el auditor tiene que mejorar es un mapa de riesgos de las distintas funciones y procesos que tiene la organización". Es importante que el auditor tenga conocimiento de las funciones de la compañía y de sus colaboradores, además de los distintos procesos que se utilizan. El mecanismo para identificar la información antes mencionada son los manuales, los cuales deben estar divulgados e implementados en la compañía a fin de que la información sea veraz y verídica. Por otro lado, estos permiten que el auditor pueda minimizar el tiempo en su ejecución, por lo que es necesario identificar cuáles son. En el documento web de Vivanco-Vergara, Manual organizacional, política, calidad, finanzas, procedimientos, técnicas, entre otros (2017), se describen algunos de los manuales que se utilizan en las organizaciones. También es preciso conocer que su aplicación e implementación se ejecutan dependiendo de la actividad económica de la compañía y se ajustan según la necesidad, lo que permite una estructura cimentada en la organización.

Es relevante que el auditor tenga presente que contamos con dichos procesos que nos facilitan la distribución en la compañía. Esto le puede ayudar como guía para verificar si los procedimientos se están realizando de la manera adecuada, según lo establecido por la empresa. Se debe tener en cuenta que si no le brindan la información necesaria o no son claros u omiten información relevante, no va a permitir que la auditoría se lleve de manera adecuada.

Para el investigador Díaz-Castro (2016), los profesionales cuentan con un buen conocimiento acerca del decreto 302 de 2015, lo que permitirá que desarrollen sus actividades acorde con las exigencias de este decreto, como lo son el conocimiento de las características de la información que constituye evidencia de auditoría, los procedimientos para obtenerla y sus métodos de selección.

Por otra parte, se ha visto que los intereses personales predominan sobre los intereses particulares; esta sería una de las respuestas a los in- 
terrogantes que surgen cuando es normal ver los incumplimientos de las grandes compañías anteriormente mencionadas con accionistas, entes reguladores, proveedores y el público en general. Cabe resaltar que el código de ética aplica para todas y cada una de las profesiones; sin embargo, lo mínimo que se espera de una persona es que actúe con moral y principios éticos ante cualquier situación.

La ética ha pasado a un segundo plano a pesar de su importancia, si retomamos la idea y el pensamiento de Kant (citado en Martínez-Fierro, 2006), que establece que "pretende haber encontrado la posibilidad de afirmar un fin, no como mero capricho subjetivo o material de un ser racional, sino un fin objetivo válido para todo ser racional". No tiene razón de ser utilizar a las personas como objetos para conseguir logros personales inclusive dañando su integridad. La ética no debe ser una imposición que se haga en el ser humano, simplemente es necesaria y nos enseña la diferencia entre el bien y el mal.

En cuanto a la responsabilidad objetividad, criterio y compromiso social, se establece el código de ética del contador, el cual tiene vigencia en todos los países. Aquí, en Colombia, se reconoce a través del decreto reglamentario 302 de 2015, que incorpora al derecho colombiano una versión del código de ética para los profesionales de la contabilidad emitido por el Consejo de Normas Internacionales de Ética para Contadores (IESBA). De esta manera se ratificó que las cuestiones éticas se rigen por unos principios y que el código acompaña el análisis de muchas situaciones que amenazan con impedir su cumplimiento. El contador público, como responsable de la elaboración de los estados financieros y los estados de resultados, debe tener total conocimiento de la norma que lo rige con el fin de actuar bajo los parámetros éticos y morales y para reiterar que la información presentada sea fidedigna, no solo porque puede ser sometido en cualquier momento a auditoría por parte de la empresa o un agente externo, sino también porque su credibilidad puede quedar en entre dicho para efectuar control y revisión y para comparar y establecer que la autenticidad de los datos es real. Cabe resaltar que las empresas acuden a una segunda opinión cuando deciden contratar firmas externas con profesionales de las ciencias económicas, como contadores públicos, revisores fiscales y/o administradores financieros; estos últimos, aunque no elaboran los informes contables, están en la capacidad de hacer el análisis a la información, tomar decisiones sobre el manejo adecuado de las inversiones con el fin de generar rentabilidad y liquidez y hacer que una empresa sea atractiva financieramente en un mercado que cada vez se hace más competitivo.

\section{Normas Internacionales de Auditoría (NIA)}

A partir del 1 de enero de 2016 se dio inicio a la implementación de las NIA en Colombia, las cuales ya habían sido aplicadas en otros países y, por lo tanto, su estandarización ha permitido que cada informe de un estado 
financiero esté moderado por una secuencia de normas de aceptación internacional, con el fin de prevenir el caos o el desorden y alcanzar una comunicación eficaz. La fiscalización y control de cada detalle contable de una empresa u organización debe realizarse siguiendo una serie de normas aceptadas internacionalmente, para ratificar su claridad y eficacia. Dentro de las 36 normas que constituyen a las NIA se establecen parámetros que buscan generar confianza en la información financiera que se presenta y fortalecer los principios objetivos, las responsabilidades y las conclusiones abarcando de manera íntegra cada uno de los procesos. Por tanto, el profesional que se encargue de realizar el control interno estará en la capacidad de emitir un dictamen donde se evidencie la evaluación objetiva y sus conclusiones. A continuación, relacionaremos las NIA desde su creación.

- NIA 240: es la normativa sobre la responsabilidad del auditor en la auditoría de los estados financieros con respecto al fraude.

- NIA 315: es la normativa sobre la responsabilidad del auditor para identificar y valorar los riesgos de incorrección material en los estados financieros.

- NIA 720: responsabilidad del auditor relacionado con otra información en documentos que contienen estados financieros auditados establecida por la IFAC (2017): "Esta NIA trata de las responsabilidades que tiene el auditor con respecto a otra información, tanto financiera como no financiera [distinta de los estados financieros y del informe de auditoría correspondiente]". Dicha información es relevante porque nos permite identificar las estadísticas que no se evidencian en los estados financieros, pero que sí están en otros documentos. "El auditor puede determinar necesario considerar otra información para soportar la credibilidad de los estados financieros" (Red Global de Conocimientos en Auditoría y Control Interno, 2016). el auditor debería tener en cuenta otra información para saber si existen inconsistencia en los estados financieros, pero esto no quiere decir que sea obligación de él incluirla en su auditoría. Esto lo determina la empresa, porque al incluir la otra información, suben los costos de honorarios y el contrato debe tener otras condiciones y especificaciones, con resultados más precisos.

Parrado-Rincón (2018) indica que la principal falla de los contadores públicos de Villavicencio con respecto al manejo de la NIA 220 es la relacionada con las responsabilidades de los dirigentes por la calidad de las auditorías.

\section{Metodología}

El enfoque sobre el cual desarrollaremos la metodología será de tipo cualitativo, el cual está orientado al descubrimiento de información y permite establecer una relación de participación y comunicación entre el 
investigador, el fenómeno investigado y los participantes del proceso.

Se realizará una reflexión que permita contrastar los hallazgos de este estudio con los obtenidos en los estudios referenciados en el marco teórico, pretendiendo formular recomendaciones de formación de profesionales con competencias integrales desde lo ético.

El presente trabajo se constituye en una investigación aplicada -documental y de campo-, la cual permite la observación en contacto directo con el objeto de estudio y el acopio de testimonios, que posibilita la confrontación de la teoría con la práctica en la búsqueda de la verdad objetiva. Esta investigación partirá de técnicas de obtención de información, ya que comenzará con un rastreo documental, el cual estará encaminado a la búsqueda bibliográfica que permita recolectar información existente sobre los trabajos realizados en nuestro país, al igual que en países latinoamericanos que hayan trabajado el tema de la ética en las auditorías.

El componente metodológico de carácter inductivo y deductivo aplicado a esta investigación está centrado en el análisis y la síntesis principalmente, ya que se pretende conocer la importancia de la ética en las auditorías.

\section{Resultados}

Las NIA surgen de la creación e implementación de las Normas Internacionales de Información Financiera (NIIF), como lo señala Leonardo (2018):
Corresponden a un conjunto único de normas legalmente exigibles y globalmente aceptadas, comprensibles y de alta calidad basado en principios claramente articulados; que requieren que los estados financieros contengan información comparable, transparente y de alta calidad, que ayude a los inversionistas, y a otros usuarios, a tomar decisiones económicas.

No en todas las empresas las NIA son exigibles, solamente aplican para las grandes empresas y algunas pymes. Es necesario hacer esta aclaración puesto que las otras empresas siguen un estándar diferente, llamado NAGAS. También se debe mencionar su importancia en el presente artículo, debido a que es una herramienta exigible para grandes empresas y algunas pymes, porque es una adaptación que se implementó en todo el mundo para poder hablar en un solo idioma y comprobar el escenario financiero de la empresa con transparencia.

Se debe recordar que las normas son muy importantes y más las que rigen a las empresas, ya que deben tener claridad y cumplirse para que se pueda realizar un análisis en los distintos países. Estas surgieron de la necesidad de los diferentes modelos de negocios, empresas o entidades que hoy existen, y por ello se hace necesario un estricto control, sin importar las fronteras. Es necesario que se evalúen todas las áreas de la empresa para identificar en qué estamos fallando. Hay dos normas que ayudan al control a nivel internacional, en especial en las finanzas de las empre- 
sas, como lo son las NIA y las NIIF, las cuales son muy importantes en el marco de la competitividad a nivel internacional, porque permiten que los proveedores y clientes conozcan nuestra situación financiera.

La ética es fundamental en todos los ámbitos de nuestra vida; es esencial implementarla en la ejecución y muestra de buenos resultados. En este orden de ideas, cómo identificamos que existen reglas que nos indican cuáles son los procesos y de qué manera se deben ejecutar. Además de las normas que regulan e identifican si se están implementando adecuadamente dichos procedimientos, también encontramos reglas que establecen los comportamientos de conducta y la responsabilidad de los contadores. Hablamos del código de ética para profesionales de la contabilidad, los encargados de realizar las auditorías. Es fundamental contar con una persona que tenga claro los principios de ética y lo importante que son en la ejecución e implementación de una auditoría y más cuando hablamos de que se va a buscar en información no financiera; debe ser transparente y congruente con el informe que realice al finalizar su auditoría.

Contar al interior de las organizaciones con personas íntegras, que sean éticas, que tengan conocimiento, conozcan las leyes que rigen y se actualicen es un buen acierto para ejercer control. Para el ejemplo anteriormente mencionado, el de Odebrecht, se pudo haber detectado durante el período de la obra del tramo II de la
Ruta del Sol las irregularidades que se venían cometiendo en la contratación. Si el inadecuado manejo de la información sigue su curso, este tema será de nunca acabar, pues las entidades regulatorias solo se pronuncian cuando la situación se sale de las manos y los medios de comunicación las hacen públicas.

Los profesionales de las ciencias económicas, específicamente en las áreas contables y financieras, atraviesan una complicada problemática social por los diferentes momentos de cambios económicos decrecientes dentro de la económica y la generación de la pérdida de los valores morales. De acuerdo con lo expuesto, se determina el valor que tiene la ética de los profesionales afines de las ciencias económicas dentro su proceso de formación de competencias integrales, donde se forma el ser de su proceder ético en el entorno social, económico y empresarial.

Es de esta manera que la revisoría fiscal es un mecanismo que tiende a cambiar la cultura de poder de las empresas y el Estado, consolidando mecanismos de los ciudadanos para regular dicho poder e intervenir para evitar que las acciones de las organizaciones lesionen la estabilidad de los territorios, sus comunidades e individuos en donde esta desarrolla su objeto social.

\section{Conclusiones}

Las Normas Internacionales de Auditoría (NIA) crearon las pautas para realizar una auditoría bien estructura- 
da y sin fronteras globales. La auditoría financiera es la que nos determina cómo está la situación actual de la empresa en la parte de los recursos económicos, entre otros, de ahí la importancia de tener conocimiento amplio y suficiente de los estados financieros. Por tal motivo, es indispensable evaluar la responsabilidad del auditor de la información financiera y no financiera, que contiene información suficiente para poder determinar irregularidades y dar un dictamen, aunque para dicha información el auditor no tenga la obligación de realizar ningún aporte, pero sí de los estados financieros; si él no hace bien su trabajo, en consecuencia, la auditoría quedaría inconclusa. Se hace un llamado de atención a los contadores, empleados y empresas para que tengan especial cuidado con la información financiera y sus documentos de apoyo, porque de ahí podemos determinar mucha información que incide en el marco de las evidencias y también para que trabajen de la mano de una ética profesional que les permita dar conceptos favorables y confiables.

El papel del profesional de las ciencias económicas como contador público o administrador financiero es fundamental en la implementación de las NIA; este busca garantizar que la información contable y financiera en pequeñas o grandes empresas esté sustentada, sea real y transparente. Por lo tanto, se estarían minimizando los riesgos que se han detectado en los últimos años en algunas de las compañías más importantes del país por el manejo inadecuado de la supervisión. Las auditorías de control interno hacen parte del buen funcionamiento de una compañía y es fundamental desligar el trabajo del auditor de los beneficios personales del gerente o de quien ostente un cargo administrativo y se vea en riesgo por un dictamen, pues, de lo contrario, se generaría un conflicto de intereses que inhabilitaría la independencia de emitir un concepto tal.

Se puede concluir que una auditoría no es solo responsabilidad del auditor. En esta cadena de información participan varias áreas de la organización, como, por ejemplo, la persona que prepara la información y la suministra y la que da el visto bueno, que es parte responsable dentro del proceso. Las implicaciones van más allá de la reputación de la organización; las NIA precisamente son normas internacionales estandarizadas que, si se llevan a cabo, fomentan la inversión extranjera y el fortalecimiento empresarial.

\section{Referencias}

¿Qué son las NIIF? (2018). Gerencie. Consultado el 25 de agosto de 2020. https://www.gerencie.com/que-son-lasniif.html

504 contadores públicos han sido sancionados por casos de corrupción, revela informe. (2018, 1 de marzo). Universidad Libre. https://bit.ly/34ZxMif

Bermúdez-Gómez H. (2018, 2 de febrero). Momentos de la regulación ética. Actualícese. https://bit.ly/31/msog

Díaz-Castro, J., Parrado-Rincón, W., Moya-Martínez, S., Saénz-Peña, K., \& Cepeda-Vigoya, M. (2016). Perfil profesional del revisor fiscal y el auditor respecto 
a la NIA 500. Revista Gestión, Organizaciones y Negocios, 3(2), 6-13. https:// doi.org/10.22579/23463910.55

Federación Internacional de Contadores. (2017). Norma internacional de auditoría 720 (revisada) responsabilidades del auditor con respecto a otra información. Auditors Censors. https://www.auditorscensors.com/uploads/20170109/ NIA_ES_720_Revisada.pdf

Grimaldo-Lozano, L. C. (2014). La importancia de las auditorías internas y externas dentro de las organizaciones [tesis de pregrado, Universidad Militar Nueva Granada]. Repositorio Institucional UMNG. https://bit.ly/2ENEf4y

La importancia del auditor interno en las organizaciones. (2019). Los Recursos Humanos. https://bit.ly/2YJPD8I

Martínez-Fierro, H. (2006). Kant: una ética para la modernidad. Revista Diálogos de Saberes, (24), 181-194. https:// dialnet.unirioja.es/descarga/articulo/2740979.pdf

NIA 240 - Responsabilidades del auditor en la auditoría de estados financieros con respecto al fraude. AOB Auditores. Consultado el 25 de agosto de 2020. https://aobauditores.com/nias/nia240/

NIA 315 - Responsabilidad del auditor para identificar y valorar riesgos. AOB Auditores. Consultado el 25 de agosto de 2020. https://aobauditores.com/nias/ nia315/

NIA 705 - Emitir un informe adecuado. AOB Auditores. Consultado el 25 de agosto de 2020. https://aobauditores. com/nias/nia705/
Nuño, P. (2017). Auditoría financiera. Emprende Pyme. https://www.emprendepyme.net/auditoria-financiera.html

Pareja, D. (2018). Estudio del caso Interbolsa: 6 años después. Riesgo Cero. https://www.riesgoscero.com/blog/estudio-del-caso-interbolsa-6-anos-despues

Parrado-Rincón, W., Gutiérrez-Ladino, E., \& Rodríguez-Ruiz, J. (2018). Norma internacional de auditoría (NIA) 220: control de calidad para auditorías de información financiera histórica y el desempeño del auditor externo. Revista Gestión, Organizaciones y Negocios, 5(1), 31-41. https://doi. org/10.22579/23463910.11

Red Global de Conocimientos en Auditoría y Control Interno. (2016). Normas Internacionales de Auditoría. Material de apoyo. Auditool. https://bit.ly/3IXAPUE

Vivanco-Vergara, M. E. (2017). Los manuales de procedimientos como herramientas de control interno de una organización. Revista Universidad y Sociedad, 9(3), 247-252. http://scielo.sld. cu/pdf/rus/v9n3/rus38317.pdf 